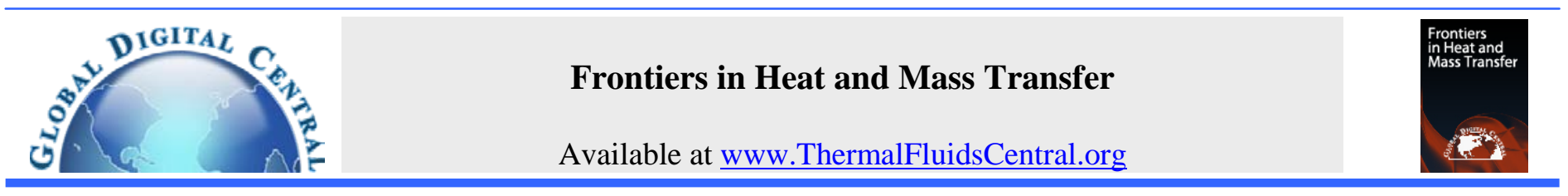

\title{
MASS TRANSFER MODELING OF HEPATIC DRUG ELIMINATION USING LOCAL VOLUME AVERAGING APPROACH
}

\author{
Mohammad Izadifar, ${ }^{\mathrm{a}, \mathrm{*}}$, Jane Alcorn ${ }^{\mathrm{c}}$ \\ ${ }^{a}$ Division of Biomedical Engineering, College of Engineering, University of Saskatchewan, Saskatoon, SK,S7N5A9 Canada \\ ${ }^{b}$ Department of Mechanical Engineering, College of Engineering, University of Saskatchewan, Saskatoon, SK,S7N5A9 Canada \\ ${ }^{c}$ Division of Pharmacy, College of Pharmacy and Nutrition ,University of Saskatchewan, Saskatoon, SK,S7N5C9 Canada
}

\begin{abstract}
Applying local volume averaging method a mathematical model including liver porosity, tortuosity, permeability, unbound drug fraction, drugplasma diffusivity, axial/radial dispersion and hepatocellular metabolism parameters was developed for hepatic drug elimination. The model was numerically solved using implicit finite difference method to describe drug concentration gradient with time across the liver. Statistically validated by observations and other models, the model suggested axial dispersion as a significant variable in drug distribution across the liver. Sensitivity analyses revealed that lower liver porosity resulted in faster drug distribution across the liver, and bioavailability was sensitive to the interaction between unbound fraction and intrinsic clearance.

Keywords: Liver, local volume averaging, mass transfer, modeling
\end{abstract}

\section{INTRODUCTION}

The liver plays a very important role in the elimination of drugs, toxic substances, and harmful biochemical products produced by the body. The liver's essential role in the maintenance of homeostasis as well as drug and toxin elimination in the body demands a detailed understanding of liver function. Mechanistic models that effectively describe liver function can play an important role in understanding and predicting drug concentration and hepatic metabolic performance. Different physiological models have been developed for the liver based on different degrees of simplifications and assumptions. The wellstirred (WS) model and the parallel-tube (PT) model are the two most commonly used models describing drug elimination by the liver (Pang and Rowland, 1977). These models are based on idealized situations of blood flow and drug distribution in the liver with an implicit assumption that the partition ratio of free drug between sinusoidal blood and hepatocytes is constant. In the well-stirred model, the drug is assumed to be instantaneously and homogeneously mixed with the blood in the liver resulting in very uniform drug concentration across the liver. Distributed parallel tube model was developed based on a distribution function defining the volumetric fraction of blood flow transported in each tube (Bass et al., 1978; Forker and Luxon, 1978). Roberts and Rowland (1986) suggested a physiological-based dispersion (DP) model based on the residence time distribution of the drug in the liver. Although the DP model takes into account hepatocellular permeability of drugs, it fails to consider the hepatic tissue partition coefficient and tissue structural characteristics such as porosity and tortuosity. Hisaka and Sugiyama (1998) solved the fundamental equation of DP at nonlinear and unsteady state hepatic elimination of substances using explicit finite difference method which is less accurate than the implicit method. They also incorporated their method into a nonlinear least-squares fitting algorithm to estimate pharmacokinetics (PK) parameters. Their numerical model was based on a series of $m$ compartments corresponding to the free or bound drug in the vascular space, blood cells, or Disse space, or in the cells at various radial distances from the vasculature; however, the model was unable to include the instantaneous equilibrium conditions (e.g.. tissue partition coefficient) between the tissue cells and the blood. In addition, the structural properties of the liver tissue (e.g. porosity, tortuosity, and permeability) were not included in the model.

Because biological tissues are formed by a porous extracellular matrix consisting of dispersed cells and connective voids saturated by an interstitial fluid, the application of porous media concepts for describing transport phenomena (e.g. blood-tissue nutrient transfer) in tissues has been attractive to researchers within the last two decades. Charles et al. (1989) introduced a three dimensional finite element model for the fluid flow and mass transfer in the liver based on the principles of porous media. Described by Darcy's law, they considered the blood flow in the liver as a creeping flow and then developed the momentum and mass transfer equations. Neglecting axial dispersion, the mass transfer governing equation was unable to describe the hepatocellular drug metabolism. Although the drug transfer from the blood to the cells was described by cell membrane permeability, the model was unable to include the partitioning of drug between tissue and blood. The lack of information of tissue partition coefficient, the axial dispersion and hepatocellular metabolism in the model were significant downsides of the proposed model.

Employing the concepts of porosity and tortuosity in porous media Nicholson (2001) demonstrated the significant effects of porosity and tortuosity on delivery rate of oxygen, glucose and drug substances from the vascular system to the brain cells. Using the porous media approach he pointed out that void fraction and tortuosity can reveal how the local geometry of the brain changes with time or under pathological

\footnotetext{
*Corresponding author.Email: mohammad.izadifar@usask.ca
} 
conditions. A porous media based continuum-mechanical model was presented by Ehlers et al. (2011) to describe the complex fluid-structure interaction in biological tissue on a macroscopic scale. They treated the biological tissue as a porous medium where cells and the extracellular matrix were treated as the solid phase, whereas extracellular and interstitial liquids and necrotic debris were the fluid phase. Their model was able to describe the growth of the tissue by a distinct mass exchange between the fluid and solid phase. They used their model for the simulation of the early stages of a vascular tumor growth in the framework of the finite element method.

Although several mechanistic models have been presented for predicting the hepatic drug elimination, an improved model can be achieved if the liver structural properties (e.g. porosity, tortuosity and permeability) together with the drug physicochemical characteristics (e.g. partition coefficient, plasma unbound fraction, affinity), non-linear hepatocellular metabolism and transport properties (e.g. molecular diffusion coefficient, dispersion coefficient, perfusion rate) are compiled to a single governing equation to spatially describe the transient drug distribution across the liver. The main objective of this study is to employ local volume averaging (LVA) method for modeling and simulation of drug elimination by the liver. In LVA method the liver is treated as a periodic structure in which the fluid phase, blood, flows through a solid matrix of cells (hepatocytes) where a local equilibrium between cells and the blood is assumed and a nonlinear hepatocellular metabolism takes place. Time dependent drug concentration gradient across the liver, drug hepatic elimination rate, hepatic clearance and bioavailability are predicted. The predictability of the model will be statistically evaluated using reported observations of seven drugs and well known models followed by performing sensitivity analyses with respect to the liver porosity and axial dispersion.

\section{THEORY}

Liver consists of a matrix of hepatocytes (liver cells) and spaces filled with fluids e.g. blood and can be treated as a porous medium. In porous media when a solid matrix cannot be described within pore size, a representative elementary volume (REV) with a characteristic length of $l$ and volume of $V_{l}$ is defined to represent the structure of the matrix. Since a lobule is functionally considered as a representative processing unit from physiological viewpoint, the liver sinusoidal can be considered as a REV based on which a fluid flow biphasic model can be developed (Hall, 2006). Since a REV is defined as the smallest differential volume resulting in statistically meaningful average properties of a porous medium, a lobule can be also considered as the REV of the liver tissue for deriving a governing mass transfer equation if certain length scales of the liver structure are satisfied.

\subsection{Mathematical Modeling}

Liver tissue is composed of spatially repeated hexagonal units consisting of hepatocytes or liver cells lining blood-filled sinusoids of diameter of $d_{p}$ (see Fig. 1a). Bloods from branches of the portal venule and hepatic arteriole are mixed and radially and axially flow through the sinusoids to subsequently drain into the central vein. Substances (e.g. oxygen, drugs) are transferred from the blood to the hepatocytes during flow through the sinusoids. A REV may be simplified as a solid matrix of hepatocytes where the fluid phase, blood, enters and leaves the volume while radially and axially distributed in the matrix with no blood flow into the solid phase (see Fig. 1b). Then, local volume averaged properties over the REV is defined as (Kaviany, 1995):

$$
\langle\varphi\rangle=\frac{1}{V_{l}} \int_{V_{l}} \varphi d V
$$

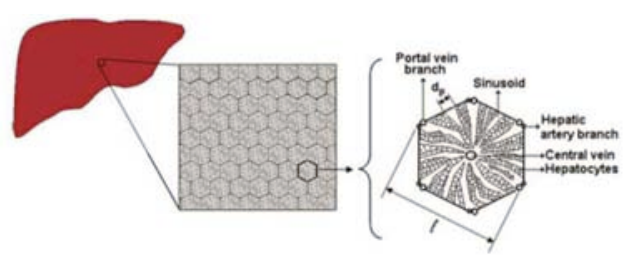

(a)

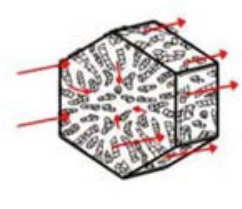

(b)
Fig. 1 Schematic diagram of the liver microstructure with associated length scales.

where $\varphi$ is the property of interest (e.g. porosity) and $\langle\varphi\rangle$ is the local volume averaged property. Averaged transport governing equations and properties over the REV is called local volume averaging (LVA) method the validity of which must be verified according to the medium length scales as:

$k_{\text {phys }}^{1 / 2}<<d_{p}<l<<L$

where $k_{\text {phys }}$ is the sinusoidal based permeability $\left(\mathrm{m}^{2}\right), d_{p}$ is the average sinusoidal diameter $(\mathrm{m}), l$ is the length scale of REV $(\mathrm{m})$, and $L$ is the length of the liver tissue $(\mathrm{m})$. Since a liver approximately consists of one million lobules (Jones and Spring-Mills, 1998), for a normal liver tissue with a volume of $1223 \pm 217 \mathrm{~cm}^{3}$ (Zhou et al., 2007), the linear dimension of a single lobule will be approximately $600 \mu \mathrm{m}$. Considering the liver sinusoidal based permeability of $3.3 \times 10^{-13} \mathrm{~m}^{2}$ (Bonfiglio et al., 2010), the sinusoid diameter of a few cells (i.e. $<60$ $\mu \mathrm{m}$ ), and the liver tissue length of $\sim 20 \mathrm{~cm}$, the validity condition of LVA is evaluated as $5.7 \times 10^{-7}<<6 \times 10^{-5}<6 \times 10^{-4}<<2 \times 10^{-1}$ which satisfies the LVA condition given in Eq. (2).

The liver geometry was simplified as a slab with the same thickness and volume as the liver (see Fig. 2). Having the liver average volume and thickness, the length of the representative slab was calculated as the equivalent length of the liver, $L$. As shown in figure $2 \mathrm{a}$, the bloods from portal venule and hepatic artery are mixed and perfused across the liver and leaves through the hepatic vein towards vena cava. Figure $2 b$ shows the porous differential element of the liver tissue with a length of $\Delta x$ over which the differential mass balance of a drug is applied. As mathematically illustrated in figure $2 \mathrm{~b}$, the drug compounds in blood flow in the liver are transported into the differential element by means of three mass transport mechanisms, molecular diffusion, axial/radial dispersion, and advection. In the same way the drug is transported out of the element by the same mass transport mechanisms.

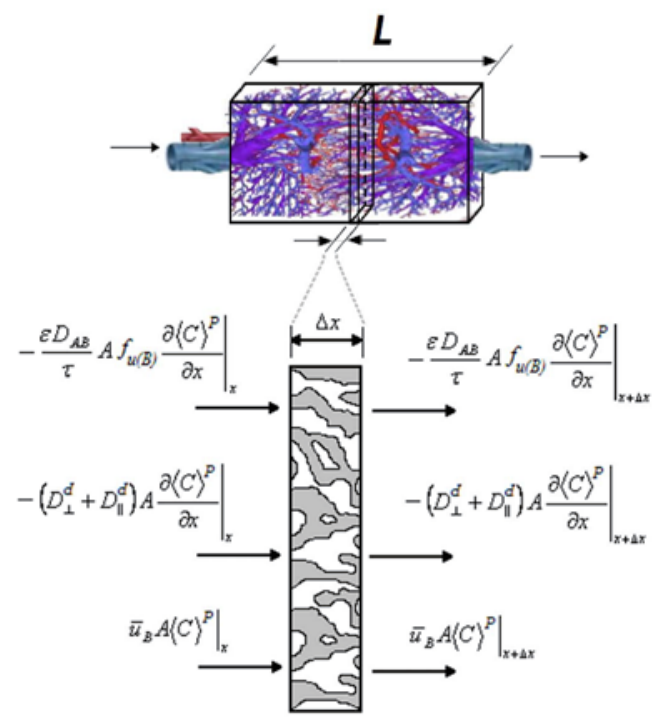

Fig. 2 Schematic diagram of the liver microstructure with associated length scales. 
As the blood flows through the sinusoids (pores of the element) with a Darcy velocity, the unbound drug in plasma is assumed to be locally in equilibrium with the hepatocytes within which the drug undergoes hepatocellular metabolism at a metabolism rate described as:

$\hat{m}_{\text {met }}=C l_{\text {int_invivo }} f_{u(B)}\langle C\rangle^{p}$

where $\hat{m}_{m e t}$ is the hepatocellular metabolism rate normalized by the liver tissue volume $\left(\mathrm{mgs}^{-1} \mathrm{ml}^{-1}\right), f_{u(B)}$ is the unbound fraction of the drug in the blood, $\mathrm{Cl}_{\text {int-in vivo }}$ is the average value of in vivo hepatic intrinsic clearance $\left(\mathrm{s}^{-1}\right)$, and $\langle C\rangle^{P}$ is the local volume averaged drug concentration in plasma $\left(\mathrm{mgml}^{-1}\right)$. In addition to Eq. (3), the concentration dependent hepatocellular metabolism can be described by Michaelis-Menten equation as:

$\hat{m}_{m e t}=\frac{M_{\max } f_{u(B)}\langle C\rangle^{P}}{K_{M}+f_{u(B)}\langle C\rangle^{P}}$

where $M_{\max }$ and $K_{M}$ are maximum metabolism rate capacity $\left(\mathrm{mgs}^{-1} \mathrm{ml}^{-1}\right.$ ) and affinity term $\left(\mathrm{mgml}^{-1}\right)$, respectively. Applying a transient mass balance over the differential element with a thickness of $\Delta x$ results in:

$$
\begin{aligned}
& \left.\left(-\frac{\varepsilon D_{A B}}{\tau} A f_{u(B)} \frac{\partial\langle C\rangle^{P}}{\partial x}\right)\right|_{x}-\left.\left(-\frac{\varepsilon D_{A B}}{\tau} A f_{u(B)} \frac{\partial\langle C\rangle^{P}}{\partial x}\right)\right|_{x+\Delta x}-m_{m e t} \\
& +\left.\left(-D_{\|}^{d} A \frac{\partial\langle C\rangle^{P}}{\partial x}\right)\right|_{x}-\left.\left(-D_{\|}^{d} A \frac{\partial\langle C\rangle^{P}}{\partial x}\right)\right|_{x+\Delta x} \\
& +\left.\bar{u}_{B} A\langle C\rangle^{P}\right|_{x}-\left.\bar{u}_{B} A\langle C\rangle^{P}\right|_{x+\Delta x}=\frac{\Delta\left((1-\varepsilon) A \Delta x K^{*} f_{u(B)}\langle C\rangle^{P}+\varepsilon A \Delta x\langle C\rangle^{P}\right)}{\Delta t}
\end{aligned}
$$

where $D_{A B}$ is the molecular diffusion of the unbound drug in the plasma $\left(\mathrm{m}^{2} \mathrm{~s}^{-1}\right), \varepsilon$ is the sinusoidal based porosity, $\tau$ is the sinusoidal based tortuosity, $f_{u(B)}$ is the unbound fraction of the drug in the blood, $A$ is the cross sectional area perpendicular to the hepatic blood flow into the liver tissue representative slab $\left(\mathrm{m}^{2}\right), x$ is the position of the drug compound in the liver (m), $m_{m e t}$ is the hepatocellular metabolism rate $\left(\mathrm{mgs}^{-1}\right), K^{*}$ is the liver tissue partition coefficient, $t$ is time (s), $D_{\|}^{d}$ is axial dispersion coefficient $\left(\mathrm{m}^{2} \mathrm{~s}^{-1}\right)$, and $\bar{u}_{B}$ is the blood Darcy velocity $\left(\mathrm{ms}^{-1}\right)$ obtained from the Darcy law given as:

$\bar{u}_{B}=\frac{Q_{h}}{A}$

where $Q_{h}$ is the hepatic perfusion rate $\left(\mathrm{mls}^{-1}\right)$. Having the sinusoidal permeability of the liver tissue and the blood pressure drop across the liver, the blood Darcy velocity can be obtained as:

$\bar{u}_{B}=-\frac{k_{\text {phys }}}{\mu} \frac{\Delta P}{L}$

where $\Delta P / L$ is the linear blood pressure gradient (Pas.m ${ }^{-1}$ ) across the liver and $\mu$ is the blood viscosity (Pas.s). Since, according to Darcy's law, the Darcy velocity of the blood is considered as the average blood velocity across the liver, the axial dispersion coefficient is assumed to be constant due to the constant Darcy velocity. Based on the dispersion number $\left(\mathrm{D}_{\mathrm{n}}=\mathrm{Pe}^{-1}\right)$ of 0.17 for the liver (Roberts and Rowland, 1986), an axial dispersion coefficient of $1.28 \times 10^{-4} \mathrm{~m}^{2} / \mathrm{s}$ was used for simulation. The partial differential governing equation of drug transfer, Eq. (8), then, is derived by substituting the metabolism term by Eq. (3) and normalizing Eq. (5) by the volume of the differential element followed by simplifying the equation and letting $\Delta x$ and $\Delta t$ go to zero as:

$\left(D_{\|}+\frac{D_{A B} \varepsilon f_{u(B)}}{\tau}\right) \frac{\partial^{2}\langle C\rangle^{P}}{\partial x^{2}}-\bar{u}_{B} \frac{\partial\langle C\rangle^{P}}{\partial x}-\hat{m}=\left(f_{u(B)}(1-\varepsilon) K^{*}+\varepsilon\right) \frac{\partial\langle C\rangle^{P}}{\partial t}$

where $\hat{m}_{m e t}$ is the hepatocellular metabolism rate normalized by the liver tissue volume $\left(\mathrm{mgml}^{-1} \mathrm{~s}^{-1}\right)$ given by Eqs. (3) and (4) for a constant and nonlinear hepatocellular metabolism, respectively. Accordingly, the intrinsic clearance function is defined as:

$C l_{\text {int }}= \begin{cases}C l_{\text {int-invivo }} & \text { if } C l_{\text {int }} \neq f(C) \\ \frac{M_{\max }}{K_{M}+f_{u(B)}\langle C\rangle^{P}} & \text { if } C l_{\text {int }}=f(C)\end{cases}$

As indicated in Eq. (8), the time dependent drug concentration in both hepatocytes and plasma is described by the accumulation term on the right side of the equation. The liver structural characteristics of porosity and tortuosity are included in the governing equation while the blood Darcy velocity and the axial dispersion coefficient takes into account the influence of the tissue permeability. If instantaneous drug distribution in the body occurs following intravenous (IV) bolus injection of drug then the ratio of the IV dose $\left(X_{0}\right)$ to the volume of distribution $\left(V_{d}\right)$ determines the plasma drug concentration at the blood entry to the liver. At the liver inlet and outlet boundaries the convective mass flow predominates and diffusion and dispersion are assumed to be insignificant. Consequently, the boundary conditions and initial conditions can be described as:

$$
\begin{aligned}
& \left\{\begin{array}{l}
\langle C\rangle^{P}(x=0, t)=\frac{X_{0}}{V_{d}} \& D_{\|}^{d}(x=0, t)=0 \\
\left.\frac{\partial\langle C\rangle^{P}}{\partial x}\right|_{x=L, t}=0 \quad \& D_{\|}^{d}(x=L, t)=0
\end{array}\right. \\
& \langle C\rangle^{P}(x, t=0)=0
\end{aligned}
$$

When the drug distribution within the liver is complete, the model can predict the plasma unbound drug concentration just at the liver outlet. With knowledge of the drug concentration at the inlet and outlet of the liver the hepatic clearance $\left(C l_{h-L V A}\right)$ can be evaluated as:

$$
C l_{h-L V A}=\frac{Q_{h}\left(\left.\left\langle C_{u}\right\rangle^{P}\right|_{x=0}-\left.\left\langle C_{u}\right\rangle^{P}\right|_{x=L}\right)}{\left.\left\langle C_{u}\right\rangle^{P}\right|_{x=0}}
$$

where $\left\langle C_{u}\right\rangle^{P}$ is the local volume averaged unbound drug concentration $\left(\mathrm{mgml}^{-1}\right), L$ is the equivalent length of the liver (m). In order to compare the proposed model to other models, the hepatic clearance was calculated for WS, PT and DP models, respectively, as follows (Ito and Houston, 2004):

$$
\begin{aligned}
C l_{h-W S} & =\frac{Q_{h} f_{u(B)} C l_{\text {int-invivo }} V_{t}}{Q_{h}+V_{t} f_{u(B)} C l_{\text {int }}} \\
C l_{h-P T}= & Q_{h}\left(1-\exp \left(-\frac{f_{u(B)} C l_{\text {int-invivo }}}{\left.\left.\frac{Q_{h}}{V_{t}}\right)\right)}\right.\right. \\
C l_{h-D P}= & Q_{h}-\frac{4 Q_{h} a}{(1+a)^{2} \exp \left(\frac{(a-1)}{2 D_{n}}\right)-(1-a)^{2} \exp \left(\frac{-(a+1)}{2 D_{n}}\right)}
\end{aligned}
$$


where $C l_{h-W S}, C l_{h-P T}$ and $C l_{h-D P}\left(\mathrm{~s}^{-1}\right)$ are the hepatic clearance suggested by WS, PT and DP models, the dispersion number of $D_{n}$ in Equation (15) is 0.17 and $a$ is defined as (Roberts and Rowland, 1986):

$$
a=\sqrt{1+\frac{4 f_{u(B)} V_{t} C l_{\text {int-invivo }} D_{n}}{Q_{h}}}
$$

where $V_{t}$ is the volume of the liver tissue $(\mathrm{ml})$ and can be calculated for males and females by Equations (17) and (18), respectively, as a function of body weight as (Anderson et al., 2000):

$$
\begin{aligned}
& V_{t}=415+17.3 W_{b}+210.5 \log \left(10 a_{c}\right) \\
& V_{t}=389+14.5 W_{b}
\end{aligned}
$$

where $V_{t}$ is the liver volume $(\mathrm{ml}), W_{b}$ is the body weight $(\mathrm{kg})$ and $a_{c}$ is the number of drinks per day. With estimates of hepatic clearance $\left(C l_{h}\right)$ and the hepatic perfusion rate $\left(Q_{h}\right)$, the bioavailability is calculated as (Roberts and Rowland, 1986):

$F_{h}=1-\frac{C l_{h}}{Q_{h}}$

\subsection{Numerical Solution of the Moldel}

The model was numerically solved based on some assumptions and physical arguments as:

- Extrahepatic clearance of the drug was negligible.

- Biliary excretion of parent drug was negligible.

- Following IV administration drug undergoes instantaneous distribution in the body such that plasma drug concentration at the liver inlet could be assumed as the ratio of IV dose to the volume of distribution of the drug.

- Blood concentration to plasma concentration ratio of the drug was unity.

- $\quad$ The unbound fraction of the drug in the blood remained unchanged with time.

- Radial dispersion was negligible compared to advection and axial dispersion as each lobule function is independent of neighboring lobules.

The liver representative slab was divided into $N+1$ nodes where $N$ was the node at the liver outlet and $N+1$ was a fictitious node just in the hepatic vein. Then, Eq. (8) was discretized using implicit finite difference method. Rearranging the finite difference equations resulted in the following system of algebraic equations as:

$C_{i}^{t}=\frac{\alpha C_{i}^{t-1}+\beta C_{i+1}^{t}+(2+\gamma) C_{x=0}^{t}}{(3+\alpha+\gamma+\theta)} \quad(\mathrm{i}=1)$

$C_{i}^{t}=\frac{\alpha C_{i}^{t-1}+\left(\frac{3 \beta}{16}\right)\left(C_{i+1}^{t}+C_{i-1}^{t}\right)+\left(\frac{\gamma}{2}\right) C_{i-1}^{t}}{\left(\alpha+\frac{3 \beta}{8}+\frac{\gamma}{2}+\theta\right)} \quad(1 \leq \mathrm{i} \leq N)$

$C_{i}^{t}=\frac{\alpha C_{i}^{t-1}-\left(\frac{3 \beta}{8}\right) C_{i-1}^{t}+\left(\frac{\gamma}{2}\right) C_{i-1}^{t}}{\left(\alpha-\frac{3 \beta}{8}+\frac{\gamma}{2}+\theta\right)} \quad(\mathrm{i}=N+1)$

where $i$ is the node index, $C$ represents the plasma unbound drug concentration, and the coefficients of $\alpha, \beta, \gamma$, and $\theta$ are defined as:

$$
\begin{gathered}
\alpha=\frac{(1-\varepsilon) K^{*}+\left(\frac{\varepsilon}{f_{u(B)}}\right)}{\Delta t}, \beta=\frac{16\left(\frac{D_{\|}^{d}}{f_{u(B)}}+\frac{D_{A B} \varepsilon}{\tau}\right)}{3 \Delta x^{2}}, \\
\gamma=\frac{2 \bar{u}_{B}}{f_{u(B)} \Delta x}, \quad \theta= \begin{cases}C l_{\text {int-invivo }} & \text { if } C l_{\text {int }} \neq f(C) \\
\frac{M_{\max }}{K_{M}+C_{i}^{t-1}} & \text { if } C l_{\text {int }}=f(C)\end{cases}
\end{gathered}
$$

The mesh size was determined based on the sensitivity analysis of the drug concentration gradient across the liver with respect to the number of nodes at $100 \mathrm{~s}$. Time step size was determined based on the analysis of stability, accuracy and the speed of solution. Gauss-Seidel iterative method with a convergence criterion of $10^{-6}$ was used for solving the system of algebraic equations simultaneously in MATLAB. Simulation was performed for seven drugs, lidocaine, metoprolol, verapamil, caffeine, timolol, diazepam, and phenacetin (Shibata et al, 2002), at a hepatic perfusion rate of $1500 \mathrm{mlmin}^{-1}$ and a sinusoidal porosity of 0.12 (Bonfiglio et al., 2010) for a time-course of $200 \mathrm{~s}$ following a $5 \mathrm{mg}$ IV dose of each drug. The tortuosity of the liver tissue was calculated according to the tortuosity of porous media consisting of layer by layer parallel rods as (Perry and Green, 2008):

$\tau=\frac{(1-\varepsilon)^{2}}{\varepsilon}$

Table 1 shows the reported pharmacokinetic properties of each drug used for the simulation. Lidocaine, with a tissue partition coefficient of 0.61 (Joseph et al., 2001), was chosen as a candidate for the simulation of drug distribution across the liver as well as for sensitivity analyses.

Table 1 Pharmacokinetic parameters ${ }^{\text {a }}$ of drugs used for simulation

\begin{tabular}{lccc}
\multicolumn{4}{c}{ Table 1 Pharmacokinetic parameters ${ }^{\mathrm{a}}$ of drugs used for simulation } \\
\hline Drug & $\begin{array}{c}\text { Unbound } \\
\text { fraction }\end{array}$ & $\begin{array}{c}\text { Intrinsic } \\
\text { clearance } \\
\left(\mathrm{mlmin}^{-1} \mathrm{~kg}^{-1}\right)\end{array}$ & $\begin{array}{c}\text { Volume of } \\
\text { distribution }\left(\mathrm{Lkg}^{-1}\right)\end{array}$ \\
\hline Lidocaine & 0.615 & 29.8 & 3.00 \\
Metoprolol & 0.883 & 17.8 & 4.5 \\
Verapamil & 0.280 & 31.0 & 4.63 \\
Caffeine & 0.650 & $1.7^{\mathrm{e}}$ & 1.06 \\
Phenacetin & 0.600 & 127.5 & 1.55 \\
Timolol & 0.400 & 7.7 & 3.5 \\
Diazepam & 0.013 & 0.3 & 1.57 \\
\hline
\end{tabular}

${ }^{\mathrm{a}}$ (Shibata et al., 2002)

\section{RESULTS AND DISCUSSION}

Based on a sensitivity analysis performed for the drug concentration gradient across the liver with respect to the grid size at $100 \mathrm{~s}$, a mesh size of $0.63 \mathrm{~mm}$ equivalent to 350 nodes was adopted for the numerical solution. According to accuracy and speed of solution, which were performed for different time step sizes of $0.1,1,5$, and $10 \mathrm{~s}$, a time step size of $1 \mathrm{~s}$ was used for the numerical solution.

Figure 3a depicts the LVA-based model predictions and observations (Shibata et al., 2002) of the hepatic clearance including the uncertainty (error bars) associated with reported data for seven drugs at a perfusion rate of $1500 \mathrm{mlmin}^{-1}$. A good agreement between predicted and reported values can be observed. Although the LVA-based model underestimates timolol and slightly overestimates caffeine hepatic clearance, the predicted values are within the uncertainties of the observed values with a coefficient of determination $\left(R^{2}\right)$ of 0.91 . Figure 3b illustrates the LVA-based model predictions and observations (Shibata et al., 2002) of bioavailability including the error bar of the reported values for seven drugs at a perfusion rate of $1500 \mathrm{mlmin}^{-1}$ for a $70 \mathrm{~kg}$ male subject. A relatively good agreement is observed between predicted and observed values although LVA-based model 
overestimates verapamil, lidocaine and timolol. The error can be attributed to the difference between observed values of oral bioavailability, which includes drug loss across the intestinal wall, and the LVA model predicted values of the hepatic bioavailability. In addition, because the error associated with the predicted hepatic clearance propagates into the predicted values of bioavailability, according to Eq. (11), the error is accumulated in the predicted bioavailability values. The influence of hepatic clearance prediction error on the calculated bioavailability can be well observed for verapamil where the underestimated hepatic clearance leads to overestimation of bioavailability.

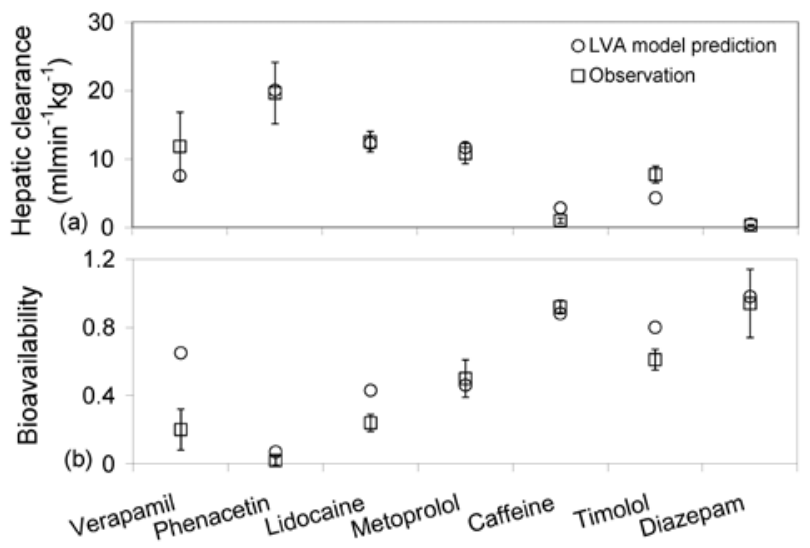

Fig. 3 Predicted values of hepatic clearance (a) and bioavailability from LVA-based model compared to observed values reported by (Shibata et al., 2002) for seven drugs.

Table 2 indicates that LVA-based model predictions of hepatic clearance at a dispersion number of 0.17 are consistent with PT and DP models; however, a larger discrepancy between LVA and WS models can be distinguished due to the significant simplifications associated with the WS model. Comparing the predicted values to the observed values, Table 2 indicates that the LVA-based model improves predictability for diazepam although the model slightly overestimates caffeine hepatic clearance compared to WS, PT and DP models.

Table 2 Observed hepatic clearance $\left(\mathrm{mlmin}^{-1} \mathrm{Kg}^{-1}\right)$ and the predicted values from LVA, WS, PT, and DP models for seven drugs

\begin{tabular}{llllll}
\hline \multicolumn{1}{c}{ Drug } & $\mathrm{Cl}_{\text {h_LVA }}$ & $\mathrm{Cl}_{\text {h_WS }}$ & $\mathrm{Cl}_{\text {h_PT }}$ & $\mathrm{Cl}_{\text {h_DP }}$ & $\begin{array}{c}\text { Observed } \\
\text { Values }^{\mathrm{a}}\end{array}$ \\
\hline Verapamil & 7.51 & 6.18 & 7.14 & 6.84 & $11.8 \pm 5.0$ \\
Phenacetin & 20.00 & 16.74 & 20.83 & 19.83 & $19.6 \pm 4.5$ \\
Lidocaine & 12.27 & 9.88 & 12.32 & 11.51 & $12.5 \pm 1.5$ \\
Metoprolol & 11.63 & 10.14 & 12.70 & 11.86 & $10.8 \pm 1.5$ \\
Caffeine & 2.83 & 1.05 & 1.08 & 1.07 & $1.0 \pm 0.4$ \\
Timolol & 4.29 & 3.11 & 3.11 & 3.35 & $7.7 \pm 1.2$ \\
Diazepam & 0.47 & 0.01 & 0.01 & 0.01 & $0.3 \pm 0.1$ \\
\hline
\end{tabular}

a (Shibata et al., 2002)

Mean squared prediction errors (MSEs) of hepatic clearance were 7.14, 18.41, 8.15, and 9.42 for LVA, WS, PT and DP models, respectively. The higher MSE in the WS model is due to its less mechanistic nature and oversimplified assumptions for the hepatic elimination process as compared with LVA, PT and DP models while MSE resulted from LVA model is close to PT and DP models because of more mechanistic assumptions associated with the models. Comparing resultant MSEs from models indicates that LVA-based model can lead to smaller MSE values of hepatic clearance. Since LVA model takes into account more structural (i.e. porosity, tortuosity) and physico-chemical parameters (i.e. tissue partition coefficient) compared to other three models, errors associated with the parameters propagate in the solution resulting in larger uncertainties in predictions. Despite the existence of such a potential error can in LVA predictions, the resultant MSE is still smaller than those of the other models which might be an indication of an improved predictability of the hepatic clearance. Another source of error in calculating MSE values is associated with the assumption of negligible extrahepatic clearance of the drugs. Any source of extrahepatic clearance associated with the reported experimental observations can increase the discrepancy between the observations and predictions which consequently leads to larger MSE. With respect to the model predictability of bioavailability, except for WS model resulting in larger MSE of 0.06, other models resulted in the same MSE value of 0.04 .

Figure 4 illustrates the plasma unbound drug concentration gradient of lidocaine across the liver at different times in the presence and absence of axial dispersion. In the absence of dispersion $\left(D_{n}=0\right)$, only diffusion and advection contributes to drug transport such that the drug distributes along the liver in $20 \mathrm{~s}$ (figure 4); however, in the presence of dispersion $\left(D_{n}=0.17\right)$ the drug is rapidly distributed in the liver such that the drug compound appears at the hepatic vein in less than $10 \mathrm{~s}$. This indicates that axial dispersion has a significant role in describing the drug distribution in the liver. This is very consistent with the results reported by Roberts and Rowland (1986) who described the residence time of a drug bolus in the liver using dispersion model.

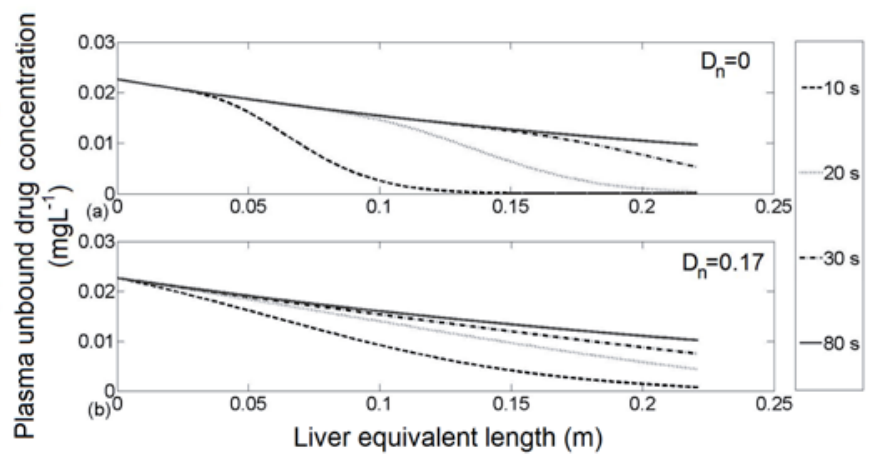

Fig. 4 Unbound drug concentration vs. equivalent length of the liver at different times following IV administration of $5 \mathrm{mg}$ lidocaine.

Figure 5 depicts the sensitivity of bioavailability to the intrinsic clearance and unbound fraction at a liver porosity of 0.12 and perfusion rate of $1500 \mathrm{mlmin}^{-1}$. For low values of unbound fraction $(<0.1)$ or intrinsic clearance $\left(<0.1 \mathrm{Lmin}^{-1} \mathrm{~kg}^{-1}\right)$ bioavailability gradually decreases with increases in either unbound fraction or intrinsic clearance or both. When unbound fraction and intrinsic clearance are low, an increase in only one does not produce a substantial drop in bioavailability due to the suppressing effect of the low value of the other variable. However, for higher values $(>0.15)$ of unbound fraction and intrinsic clearance, an increase in either one causes a significant drop in bioavailability implying that metabolism rate is sufficiently sensitive to the magnitude of values of either unbound dug fraction or intrinsic clearance.

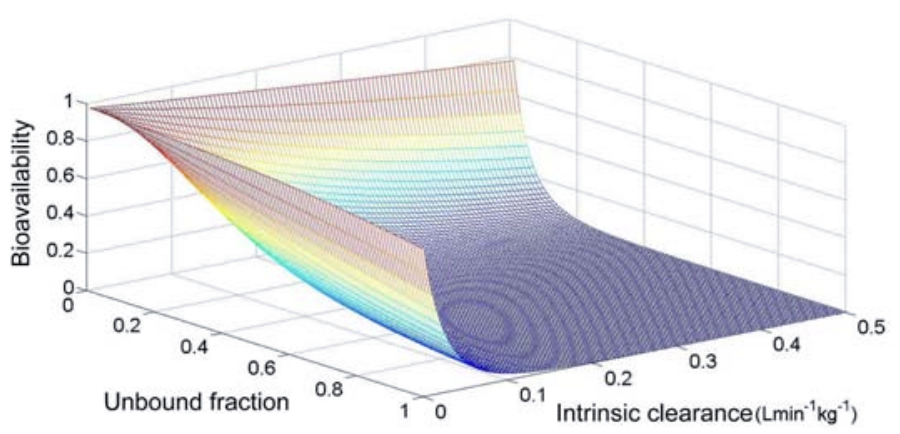

Fig. 5 Bioavailability of lidocaine versus intrinsic clearance and unbound fraction at a sinusoidal perfusion rate of $1500 \mathrm{mlmin}^{-1}$. 
Since the liver porosity may vary due to different circumstances (i.e. individual variability, disease), the sensitivity of drug concentration gradient across the liver with respect to the liver porosity was investigated. Figure 6 depicts the drug concentration gradient across the liver at different times for the axial dispersion number of 0.17 within the porosity variation limit of $0.12 \pm 5 \%$ (0.06 and 0.18 ). An increase and decrease in the liver porosity lead to higher and lower plasma drug concentration across the liver which is associated with the effect of the porosity on the sinusoidal blood velocity (pore velocity), which is obtained by the ratio of blood Darcy velocity to the porosity. Lower sinusoidal porosity leads to higher pore velocity enhancing the effect of the advection term in Eq. (8). Larger advection causes the drug to move forward faster so that the residence time is reduced and the drug concentration gradient shifts forward at any position during the transient distribution time. Higher porosity reduces the plasma drug concentration shifting the drug concentration curve backward. However, the effect of porosity on the concentration gradient becomes insignificant at $80 \mathrm{~s}$ when the drug distribution across the liver has reached steady state.

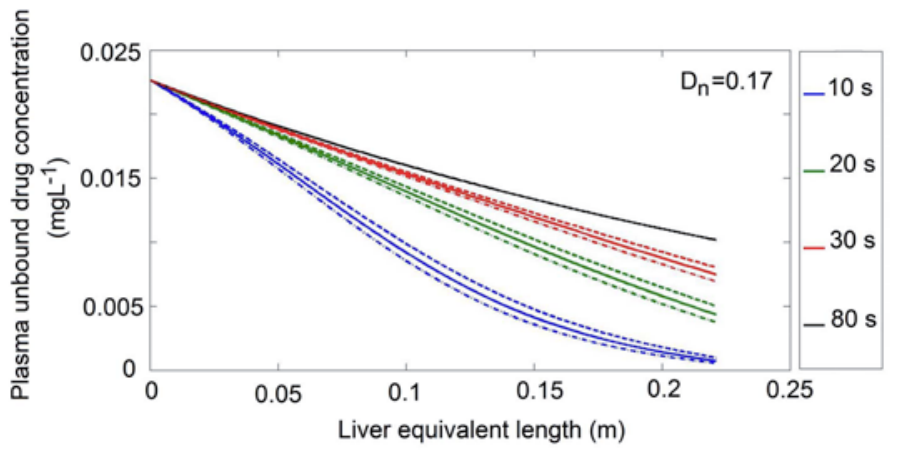

Fig. 6 The influence of porosity on plasma unbound drug concentration gradient across the liver at different times for lidocaine for axial dispersion number $\left(D_{n}\right)$ of 0.17 : - -, $\varepsilon=0.06$; - , $\varepsilon=0.12$; -.-, $\varepsilon=0.18$.

\section{CONCLUSION}

Being consistent with well know models and observations of hepatic clearance of seven drugs, the proposed LVA-based model can be successfully used for predicting transient drug concentration gradient across the liver and hepatic clearance. The sensitivity analyses revealed that the influence of axial dispersion on the drug concentration gradient and residence time of the drug across the liver is so significant that ignoring the axial dispersion causes a significant error in predicting hepatic drug elimination. The model's ability to spatially describe the time dependent drug concentration across the liver makes the model potentially useful for investigating the effect of a pathological deficiency (i.e. shunting) on the pattern of drug distribution across the liver. Future refinements of the model will address the incorporation of mass transfer resistance associated with transporters and mass diffusion across the hepatocellular membranes. A departure from reported observations may exist if the diffusion mass transfer resistance across the hepatocellular membrane is higher than convection mass transfer resistance. This situation violates the assumption of local equilibrium and can result in significant error in predictions. The assumption of local equilibrium represented by the tissue partition coefficient also precludes addition of the role of transporters and their potential contribution as a rate-limiting process in the drug intrinsic clearance.

\section{ACKNOWLEDGMENT}

The authors appreciate Dr. Oon-Doo Baik for his editorial comments and his financial support.

\section{NOMENCLATURE}

A

a

C

C

$<$

Cl

$D$

$D_{\text {| }}$

d

F

f

K

$K^{*}$

k

$L$

l

$M$

$m$

$\hat{m}$

$P$

$P e$

$Q$

$t$

$u$

V

W

$X$

$x$

Greek Symbols

$\alpha \quad$ coefficient in Eqs. (20)-(22)

$\beta \quad$ coefficient in Eqs. (20)-(22)

$\gamma \quad$ coefficient in Eqs. (20)-(22)

$\varepsilon \quad$ porosity (dimensionless)

$\theta \quad$ coefficient in Eqs. (20)-(22)

$\mu \quad$ viscosity (Pa.s)

$\tau \quad$ tortuosity (dimensionless)

$\varphi \quad$ propert of interest

$<\varphi>\quad$ local volume average of the property

Superscripts

$\begin{array}{ll}d & \text { dispersion } \\ p & \text { plasma } \\ t & \text { time index }\end{array}$

Subscripts

$A B \quad$ molecular diffusion of $\mathrm{A}$ in $\mathrm{B}$

$B \quad$ blood

h hepatic

$h \_D P \quad$ hepatic clearance associated with DP model

$h \_L V A \quad$ hepatic clearance associated with LVA model

h_PT hepatic clearance associated with PT model

$h \_W S \quad$ hepatic clearance associated with WS model

$i$

int intrinsic

int_invivo in vivo intrinsic

$o \quad$ initial

l REV length

m maximum

M associated with Michaelis_Menthon equation

met metabolism

$n \quad$ number

$p \quad$ pore

phys physiological

$u(B) \quad$ unbound drug in the blood 


\section{REFERENCES}

Anderson, V., Sonne, J., Sletting, S., Prip, A., 2000, "The volume of the liver in patients correlates to body weight and alcohol consumption," Alcohol \& Alcoholism,35, 531-532. http://dx.doi.org/10.1093/alcalc/35.5.531

Bass, L., Robinson, P.J., Bracken, A.J., 1978, "Hepatic elimination of flow substances: The distributed model,” J Theor Biol 72,161-184.

Bonfiglio, A., Leungchavaphongse, K., Repetto, R., Siggers, J.H., 2010, "Mathematical modeling of the circulation in the liver lobule," $J$ Biomech Eng 132, 1110111- 11101110. http://dx.doi.org/10.1115/1.4002563

Charles, Y., Lee, C., Rubinsky, B.,1989, “A multi-dimensional model of momentum and mass transfer in the liver," Int J Heat Mass Transfer 32, 2421-2434.

http://dx.doi.org/10.1016/0017-9310(89)90202-0

Ehlers, W., Krause, R., Markert, B., "Modelling and remodelling of biological tissue in the framework of continuum biomechanics," Proc Appl Math Mech 11, 35-38.

http://dx.doi.org/10.1002/pamm.201110010

Forker, E.L., Luxon, B., 1978, "Hepatic transport kinetics and plasma disappearance curves: Distributed modelling versus conventional approach,” Am J Physiol 235,648-660.

Hall, C., 2006, Regulation der hepatischen Mikrozirkulation bei schrittweiser Resektion im Rattenlebermodell, $\mathrm{PhD}$ dissertation, Medizinische Fakultät der Universität Duisburg-Essen, Zentrum für. Chirurgie Klinikum für Allgemeinchirurgie, Viszeral- und Transplantationschirurgie.

Hisaka, A., Sugiyama, Y., 1998, “Analysis of nonlinear and nonsteady state hepatic extraction with the dispersion model using the finite difference method,” J Pharmacokinet Biopharm 26, 495-519.

Ito, K., Houston, B., 2004, “Comparison of the use of liver models for predicting drug clearance using in vitro kinetic data from hepatic microsomes and isolated hepatocytes," Pharm Res 2,785-792. http://dx.doi.org/10.1023/B:PHAM.0000026429.12114.7d

Jones, L., Spring-Mills, E., 1988, The liver and gall bladder, $6^{\text {th }}$ ed. Urban \& Schwarzenberg Baltimore, MA.
Joseph, A.G., Jürgen, V., Joseph, P.O., 2001, "Prediction of lidocaine tissue concentrations following different dose regimes during cardiac arrest using a physiologically based pharmacokinetic model," Resuscitation 50,331-340.

http://dx.doi.org/10.1016/S0300-9572(01)00355-0

Kaviany, M., 1995, Principles of heat transfer in porous media, $2^{\text {nd }}$ ed. Springer, New York.. http://dx.doi.org/10.1007/978-1-4612-4254-3

Nicholson, C., 2001, "Diffusion and related transport mechanism in brain tissue," Rep Prog Phys 64, 815-884. http://dx.doi.org/10.1088/0034-4885/64/7/202

Pang, K.S., Rowland, M., 1977, “Theoretical considerations of a wellstirred model and a parallel tube model: Influence of hepatic blood flow, plasma and blood cell binding and hepatocellular enzymatic activity on hepatic drug clearance," J Pharmacokin Biopharm 5,625653.

http://dx.doi.org/10.1007/BF01059688

Perry, R.H., Green, D.W., 2008, Perry's chemical engineering handbook, $8^{\text {th }}$ ed. McGraw-Hill New York.

Roberts, M.S., Rowland, M., 1986, “Correlation between in-vitro microsomal enzyme activity and whole organ hepatic elimination kinetics: analysis with a dispersion model," J Pharm Pharmacol 38,177-181.

http://dx.doi.org/10.1111/j.2042-7158.1986.tb04540.x

Roberts, M.S., Rowland, M., 1986, “A Dispersion Model of Hepatic Elimination: 1. Formulation of the Model and Bolus Considerations," $J$ Pharmacokin Biopharm 5, 227-260. http://dx.doi.org/10.1007/BF01106706

Shibata, Y., Takahashi, H., Chiba, M., Ishii, Y., 2002, "Prediction of hepatic clearance and availability by cryopreserved human hepatocytes: an application of serum incubation method," ASPET 30, 892-896.

Zhou, X., Lu, T., Wei, Y., Chen, X., 2007, "Liver Volume Variation in Patients with Virus-Induced Cirrhosis: Findings on MDCT,” $A m J$ Roentgenology 189, 153-159.

http://dx.doi.org/10.2214/AJR.07.2181 